\title{
Coherent Scene Generation for Surgical Simulators
}

\author{
Raimundo Sierra ${ }^{1}$, Michael Bajka ${ }^{2}$, Celalettin Karadogan ${ }^{1}$, Gábor Székely ${ }^{1}$, \\ and Matthias Harders ${ }^{1}$ \\ 1 Computer Vision Group, ETH Zürich, Switzerland, \\ \{rsierra, szekely, mharders\}@vision.ee.ethz.ch \\ 2 Clinic of Gynecology, Dept. OB/GYN, University Hospital of Zürich, Switzerland
}

\begin{abstract}
The idea of using computer-based surgical simulators for training of prospective surgeons has been a topic of research for more than a decade. However, surgical simulation is still far from being included into the medical curriculum. Still open questions are the level of simulation realism which is needed for effective learning, the identification of surgical skill components which are to be trained, as well as the validation of the training effect. We are striving to address these problems with a new generation of highly realistic simulators. A key element of realism is the variable training scene, reflecting differences in individual patients. In this paper we describe the complete generation process of these case-by-case scenarios.
\end{abstract}

\section{Introduction}

Endoscopic interventions have become a very popular technique in surgical care. However, performing operations under these conditions demands very specific skills that can only be gained with extensive training. Virtual reality (VR) surgery simulation is one possible tool for acquiring these skills. A wide range of VR simulator systems has been proposed and implemented in recent years. Several academic projects have been carried out, for instance related to diagnostic endoscopic investigations [13], laparoscopic surgical procedures [6,1], arthroscopic interventions [14], eye surgery [9] or radiological [4] procedures. Apart from this non-exhaustive list, a growing number of commercial products is available on the market. Nonetheless, surgical simulation is still far from being included into the medical curriculum.

This is in strong contrast to the field of flight simulation. These simulator setups have been formally integrated into pilot education and are fully accepted as a training tool. Official certification rules, for instance by the FAA (Federal Aviation Administration), have been issued and pilots are allowed to register simulation hours as flight time. Also, it should be noted, that the success story of flight simulation has been independent of the often extremely high system costs.

Currently, two main difficulties can be identified hampering further advancements in the field of surgical simulation. Firstly, the lack of a clearly defined 
relationship between the training of respective surgical skills and the appropriate level of simulation realism. Secondly, the lack of appropriate assessment tools of surgical skills impeding evaluation procedures for existing setups.

In the former area, we face the still open question of the necessary level of realism to achieve a specific training effect. This point cannot be answered in general, since it depends on the skill which should be trained. Placing clips on a tube might be rehearsed on non-realistic models (and it might be even more cost-effective to train this task on real models without using a simulator at all), however, problem management in cases of unforeseen complications during surgery probably needs high realism to achieve the desired training effect.

In the latter area, we face a methodological problem. The only currently available gold standard test for effectiveness of surgical simulator training is a surgeon's performance on real patients. This situation is clearly unsatisfactory, since on the one hand it again requires patient involvement, which surgical simulation actually tries to avoid, and on the other hand, one has to face an uncontrollable environment, which specifically obstructs objective assessments. Therefore, new ways of objective and quantifiable performance measurements have to be found.

We try to tackle both of these prevalent drawbacks with the development of a reference surgical simulator of highest possible realism. In order to investigate the relationship between the degree of realism and training effectiveness, the fidelity of the high-end system will be gradually reduced. Furthermore, the highend setup will serve as a benchmark for quantifying the training effect of existing simulators. In order to meet these ambitious goals, we have to explore and extend current limits of realism in surgical simulation. It has to be noted, that in this scenario cost-effectiveness will not be of central importance.

While first attempts to achieve a high degree of realism have already been made by our group within the framework of an earlier project [12], we currently continue these endeavors within the larger scope of a Swiss National Center of Competence in Research on Computer Aided and Image Guided Medical Interventions (NCCR CO-ME, http://co-me.ch). Currently we concentrate on the simulation of hysteroscopic interventions. This work however, can be regarded as a driving application providing a prototypical testbed to investigate the generic problems discussed above in the context of a specific clinical application.

A key element for providing the fidelity needed for effective training is the definition of variable training scenarios. Just like in flight simulation, where different weather conditions, airports, system malfunctions, etc. can be defined, surgical simulation also needs the same breadth of configurable training conditions. In currently existing simulators this point is usually neglected. Single static organ models are used to build surgical scenes. Geometries are derived from MRI datasets, based on the Visible Human Project, or are artificially created with CAD systems. However, repeated training with the same single organ model will obscure training effects, since the user will adapt to this special anatomy. To acquire surgical skills, it is highly desirable that the training scene is different from 
session to session - just like in real practice. In the following we will describe the complete process we developed for defining variable training cases.

\section{Definition of Variable Training Scenarios}

Different milestones can be identified for the successful implementation of a framework for variable scene generation. To start with, a thorough understanding of the medical background related to the surgical site and the interventions which should be trained is crucial. This step includes learning of the anatomical basis, site visits in the operation theater as well as education by the medical partners. Only by doing so, is it possible to deduce the common cases encountered in clinical practice and the associated procedures. In a second step, the requirements of the simulation core on the generated scene have to be formulated. Obviously, this process has to be iterated as more features are added to the simulator. Nevertheless, the definition of the output is required for the last step in order to devise a consistent processing pipeline.

Figure 1 shows the main pipeline we propose for the instantiation of variable surgical scenes. The first two steps can be referred to as model generation and are described in Section 2.1. Next, the raw surface model has to be optimized according to the specifications of the final simulator framework. Moreover, additional components like textures and biomechanical properties are added to build a coherent surgical scene. These steps are presented in Sections 2.2 and 2.3. Justifications for the chosen sequence are given in the respective Sections. Finally, the scene can be loaded into the simulator and used for training.

\begin{tabular}{|c|c|c|c|c|}
\hline $\begin{array}{l}\text { Organ } \\
\text { Model }\end{array}$ & $\begin{array}{c}\text { Pathology } \\
\text { Model }\end{array}$ & $\begin{array}{c}\text { Mesh } \\
\text { Optimization }\end{array}$ & $\begin{array}{l}\text { Textures, } \\
\text { Biomechanical } \\
\text { Properties, ... }\end{array}$ & Simulation \\
\hline
\end{tabular}

Fig. 1. Processing pipeline for the generation of variable surgical scenes.

\subsection{Anatomical Model Generation and Pathology Integration}

One goal of our research is to generate anatomical models considering the natural variability of the healthy anatomy and to seamlessly integrate a wide spectrum of different pathologies according to the specifications from physicians. We follow a twofold strategy to alleviate the current unsatisfactory situation: Statistical modeling of healthy anatomy to capture macroscopic variability in individual patients and artificial tissue genesis to encode anatomical details and pathologies.

Since organs of any two patients will never be alike, we use statistical anatomical models to handle the variability of healthy human anatomy [2]. Parameters like age, size or weight of a patient have often a large influence on the shape of an organ. In order to generate the models, medical patient data have been 
collected and are being segmented. After creating the statistical model of the healthy organs, patient parameters can be specified to replicate a new organ geometry.

The other requirement for a reasonably realistic surgical simulator is the ability to include a wide variety of pathological cases. The large number of possible pathologies as well as the enormous range of their manifestations makes a similar statistical approach unreasonable if not impossible. Up to now, our research addressed tumor development as exemplary pathology. Three different models for the generation of tumors were successfully developed [10]. First, a cellular automaton was presented that is able to simulate the growth of uterine leiomyomas. In the next approach, a purely geometrical, CAD-based algorithm was proposed, which artificially creates myomas by defining object skeletons and subsequently revolving contour curves. Finally, particle-based models were implemented to integrate additional physiological information in the tumor growth process. Two examples of artificially generated pathologies are depicted in Figure 2. Animations and additional examples of artificially generated pathologies are available online ${ }^{1}$.
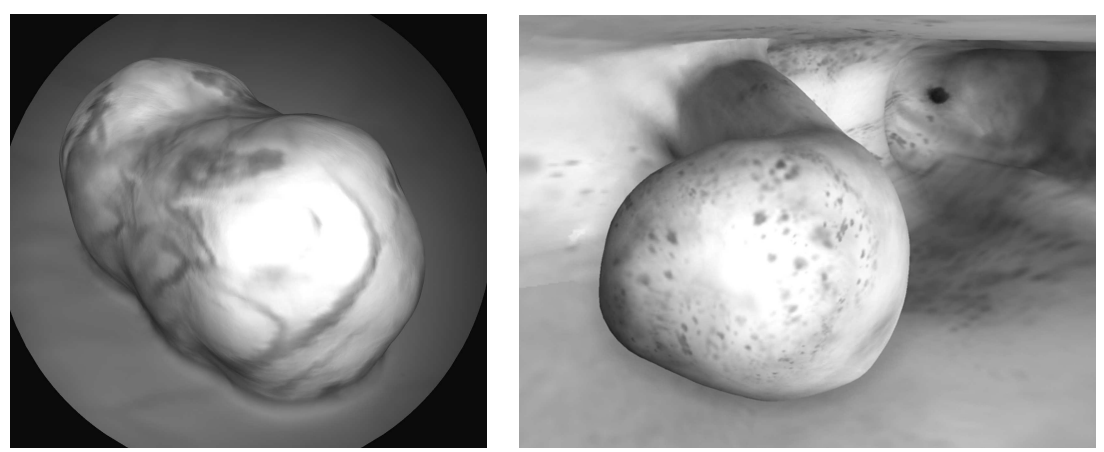

Fig. 2. Two example pathologies: Left a myoma artificially created with a cellular automaton and right an artificial polyp created with a particle system.

As shown in Figure 1 we first derive a new instance of the healthy anatomy to create a new scene. In a second step the training supervisor can then integrate different pathology models into the healthy organ, according to the training session's objective. Depending on the chosen approach, an additional step to merge the pathology and the organ is required, as has been reported earlier [10]. In any case, the pathology model has to adapt to the healthy organ, as naturally all pathologies emerge in existing organs and thus are related to the actual shape of the surrounding they are located in. The particle based pathology growth model follows strictly this biological causality.

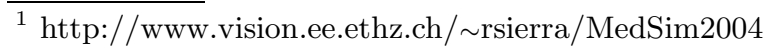




\subsection{Optimal 3D Mesh Generation}

In order to enable an individual scene generation, the resulting geometry has to be meshed fully automatically. Currently this is only possible for tetrahedral meshes. The need for stable and accurate simulation poses, however, strong constraints on the quality of the mesh. These requirements arise from the different modules involved, e.g. collision detection, collision handling as well as interactive scene modifications such as cutting. In general all modules prefer regular 3D meshes for better stability. Regular tetrahedral meshes that fill the entire tissue mass can provide the necessary base for all simulation purposes defined so far. In addition, a trade-off between accuracy, i.e. realism, and speed, i.e. computational power, has to be made.

The generated meshes so far have unpredictable properties and usually do not fullfil the requirements stated above. While the derivation of healthy organ instances can in principle incorporate mesh property criteria such as mesh and triangle sizes and restrictions on the quality like the regularity of tetrahedra, this is not possible for the generated pathologies. All approaches reported so far produce highly irregular meshes in terms of triangle sizes and connectivity, which also differ from generated instance to instance. We propose a particle based mesh generation procedure that allows us to obtain optimal meshes under the given constraints for arbitrary models.

Many methods have been proposed for the generation of tetrahedral meshes and some freely available implementations can be found online ${ }^{2}$. As most approaches rely on the provided surface triangulation and extend it into three dimensions, they cannot be used directly for optimal meshing of our models. We therefore propose to position particles in an optimal configuration both on the surface and inside the model. The user can adapt to the available computational power by regulating the density of the random particle distribution. In a second step, these particles are then interconnected to build the tetrahedral mesh.

First an augmented voxel space, i.e. an encapsulating grid over the given surface is defined. This allows for the separation of different voxel-domains: inner, outer and surface voxels. For computational speed, all voxels within the bounding box of any triangle are labeled as surface voxels and associated with the respective triangles. Since this procedure overestimates the number of surface voxels, in a second step all labeled voxels are tested against each triangle they are associated with, in order to exclude those voxels which do not actually touch or intersect a triangle. Next, the voxels outside the surface are labeled by a region growing algorithm over the 26 neighborhood of every voxel. Finally all remaining voxels can be assigned to the set of inner voxels.

A predefined number of particles is now inserted into every inner or surface voxel at random positions within the voxel. The particles in the surface voxels have to be projected onto the closest triangle within the voxel, which can be computed effectively, as the association between triangles and voxels has been established during the labeling step.

\footnotetext{
${ }^{2}$ http://www.andrew.cmu.edu/user/sowen/survey/index.html, 2.2004
} 
Finally, the particles positions are optimized to build a regular distribution over both the inner and surface domain. Therefore, the particles are rearranged based on the distance to their neighbors, similar to the method reported in the particle based tumor growth model [11]. The particles repulse or attract each other depending on an interaction profile function that relates the inter-particle distance to a force amplitude.

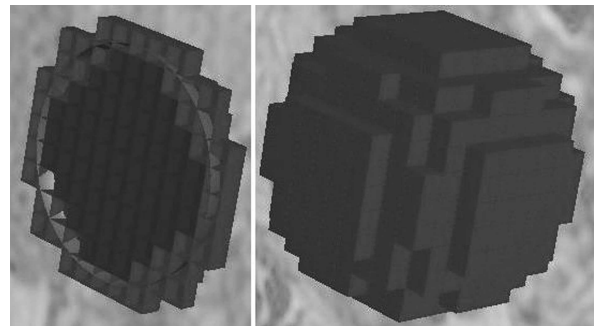

(a) Different voxel domains generated.

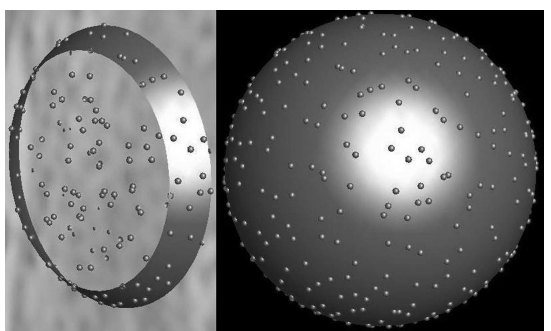

(c) Projection of particles onto the surface.

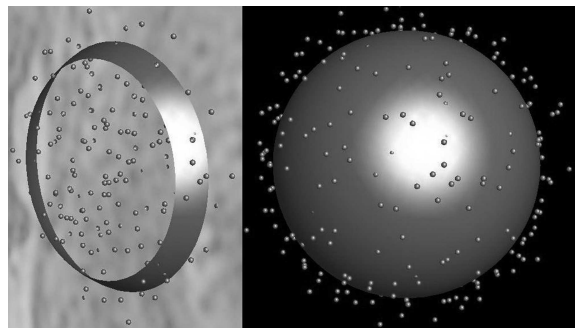

(b) Initial random distribution of particles.

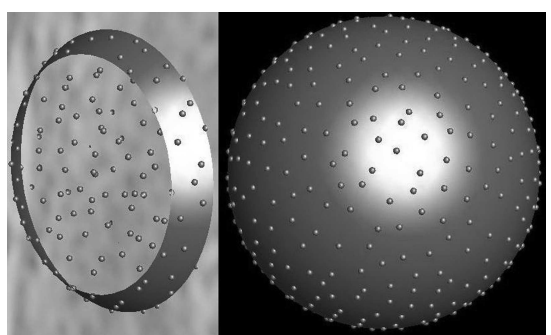

(d) Optimized particle positions.

Fig. 3. Particle distribution process. Each figure shows a $3 \mathrm{D}$ view and a crosssection near the center of the sphere.

In the course of repositioning, measures should be taken to keep inner particles inside and surface particles on the surface: surface particles are thus restricted to move only tangentially to the surface at their point of residence. Furthermore, new positions of the inner particles are restricted within the region defined by inner voxels and that of surface particles by surface voxels. Finally, the surface particles are shifted back on the surface as previously described. In order to achieve a stable and regular distribution this process is iterated and the motility of the particles is reduced in every iteration. The described process is illustrated in Figure 3, where one particle was inserted in every surface voxel and two particles in every inner voxel.

An advantage of the presented approach is the globally optimal distribution of the particles over the original surface at an arbitrary, user defined resolution. Different tetrahedralization methods like the Delaunay tessellation [3] or the 
advancing front method [7] are currently being evaluated for their feasibility to generate the final mesh based on the resulting point set.

\subsection{Photo-Realistic Texturing}

Providing correct visual information is indispensable in surgical simulation if a realistic training environment is required. A central point is the texture generation for healthy and pathologically altered organs. Correct visualization of pathologies is of central importance for our configurable anatomical models, since the visual appearance of diseased organs often differs substantially from the healthy case.

In order to solve these problems, automatic texture generation methods based on intra-operative images have been developed and pathology databases have been created. Statistical as well as procedural texturing methods were investigated by our group [8]. Organ specific base textures, i.e. textures without blood vessels, can be computed automatically by means of a texture analysis/synthesis process. Further details can then be added to the base texture by procedural methods. Figure 4 shows some earlier results of real organ textures and their artificial counterparts. We propose to generate the textures after the mesh optimization to avoid additional steps that are otherwise required to map the textures onto different meshes. Labels attached to the primary models, i.e. contact points of pathology and organ, can easily be carried along the pipeline. Since we are in control of the complete growth process of the pathologies, this information can also be used to adapt the organ textures.
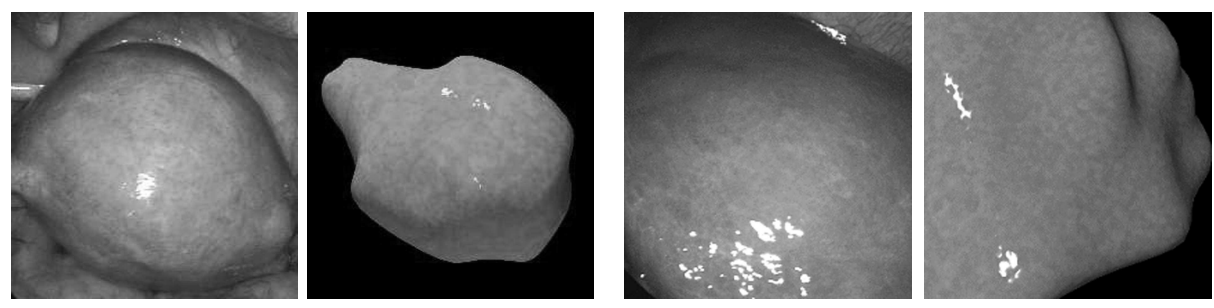

Fig. 4. Organ texture generation - Real organs during surgery and their artificially created counterparts (based on the real images) are depicted.

\section{Simulator Prototype}

Apart from the elements discussed above, further aspects of a realistic simulator are addressed within the CO-ME network. These include tissue parameter estimation, soft tissue deformation, collision detection and response, as well as force feedback devices. 
Although these points are not covered in detail in this paper, they represent important elements in a simulator setup. Especially, the tissue parameter estimation is often neglected in current systems, as realistic deformation computation cannot be performed without a knowledge of the elastic properties of living tissue. Even the best description of the mechanical behavior is useless if its parameters cannot be determined. Because significant differences exist between the mechanical properties of dead and living as well as human and animal tissue, measurements have to be performed in-vivo on patients during interventions. Special methods for this have been developed in our earlier work [5] and are currently extended.

A further fundamental element of a simulator setup is the haptic feedback. A force feedback device specifically adapted to emulate a hysteroscope is currently being developed. It provides four degrees-of-freedom, spans the appropriate workspace and generates forces in the range according to clinical specifications. An important need of the surgical procedure is the ability to exchange different endoscopic tools during interventions. This requirement is also addressed in the current hardware setup.

The first components of the hysteroscopy simulator have been combined in an early prototype displaying the initial functionality. We take advantage of a specialized supercomputing environment built on the Visual Grid ${ }^{\mathrm{TM}}$ solution of Sun Microsystems ${ }^{\mathrm{TM}}$, which has been installed within the frame of a collaborative Center of Excellence for Biomedical Simulation.

It has to be noted, that this intermediate version is far from our envisioned system for the end of the first phase of our project in summer 2005. This current prototype only demonstrates the initial framework developed for the simulator - Figure 5 shows the hysteroscope interface and a snapshot of the simulation, showing the hydrometra and a polyp inside of the uterine cavity.
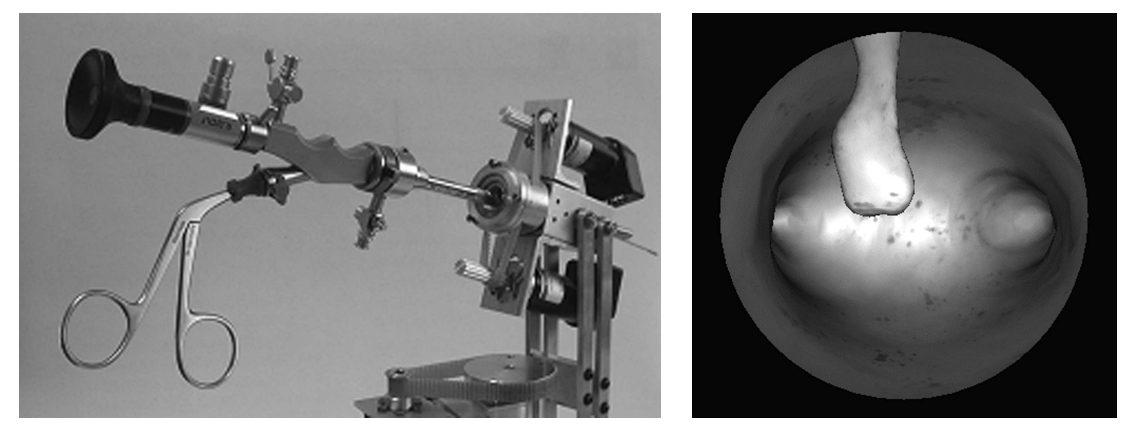

Fig. 5. Hysteroscopy simulator prototype. The haptic interface as well as a snapshot of the simulation are displayed. 


\section{Conclusion}

This report highlights the research directions we are following to develop a highly realistic simulator for hysteroscopy. The processing pipeline for the generation of variable surgical scenes has been described and first results for the generation of tetrahedral meshes suitable for simulation purposes were shown. We are currently in the process of defining interfaces for the different modules of the simulator and implementing a prototype that takes advantage of our supercomputing hardware using multiple CPUs and GPUs.

\section{Acknowledgment}

This research has been supported by the NCCR CO-ME of the Swiss National Science Foundation. We would like to acknowledge the work of Ulrich Spälter, Matthias Teschner, Stephan Weiss, Markus Grassi and Volker Meier, who contributed to the simulator prototype. We also would like to thank Sun Microsystems $^{\mathrm{TM}}$ for their support in establishing the Center of Excellence.

\section{References}

1. C. Baur, D. Guzzoni, and O. Georg. Virgy: A Virtual Reality and Force Feedback Based Endoscopy Surgery Simulator. In Proc.MMVR'98, pages 110-116, 1998.

2. T. Cootes et al. Active Shape Models - Their Training and Application. Computer Vision and Image Understanding, 61(1):38-59, 1995.

3. Q. Du and D. Wang. Tetrahedral mesh generation and optimization based on centroidal Voronoi tesselations. International Journal for Numberical Methods in Engineering, 56:1355-1373, 2003.

4. J. Hahn, R. Kaufman, A. Winick, T. Carleton, Y. Park, K.-M. O. R. Lindeman, N. Al-Ghreimil, R. Walsh, M. Loew, J. Gerber, and S. Sankar. Training Environment for Inferior Vena Caval Filter Placement. In Proc. MMVR'98, pages 291-297, 1998.

5. M. Kauer, V. Vuskovic, J. Dual, G. Szekely, and M. Bajka. Inverse finite element characterization of soft tissues. Medical Image Analysis, 6(3):275-287, 2002.

6. U. Kühnapfel, H. Krumm, C. Kuhn, M. Hübner, and B. Neisius. Endosurgery Simulations with KISMET: A flexible tool for Surgical Instrument Design, Operation Room Planning and VR Technology based Abdominal Surgery Training. In Proc. Virtual reality World'95, pages 165-171, 1995.

7. R. Lohner. Progress in grid generation via the advancing front technique. Engineering with Computers, 12:186-210, 1996.

8. V. Meier. Realistic visualization of abdominal organs and its application in laparoscopic surgery simulation. PhD thesis, ETH Zurich, 1999.

9. M. Schill, C. Wagner, M. Hennen, H. Bender, and R. Maenner. Eyesi - A simulator for intra-ocular surgery. In Proc. MICCAI'99, pages 1166-1174, 1999.

10. R. Sierra, M. Bajka, and G. Székely. Evaluation of Different Pathology Generation Strategies for Surgical Training Simulators. In Proceedings CARS, 2003.

11. R. Sierra, M. Bajka, and G. Székely. Pathology Growth Model Based on Particles. MICCAI, 1:25-32, 2003. 
12. G. Székely, C. Brechbühler, J. Dual, et al. Virtual Reality-Based Simulation of Endoscopic Surgery. In Presence, volume 9, pages 310-333, Massachusetts Insitute of Technology, June 2000.

13. D. Vining. Virtual Endoscopy: Is It Reality. In Radiology, pages 30-31, 1996.

14. R. Ziegler, W. Mueller, G. Fischer, and M. Goebel. A Virtual Reality Medical Training System. In Proc. $1^{\text {st }}$ In. Conf. on Comp. Vision, Virtual Reality and Robotics in Medicine, CVRMed'95, pages 282-286, 1995. 